\title{
Enhanced Human Body Fall Detection Utilizing Advanced Classification of Video and Motion Perceptual Components
}

\author{
Charalampos Doukas ${ }^{1}$, Ilias Maglogiannis ${ }^{2}$, Nikos Katsarakis ${ }^{3}$, Aristodimos \\ Pneumatikakis $^{3}$
}

\author{
1University of the Aegean \\ 2University of Central Greece \\ 3Athens Information Technology
}

\begin{abstract}
The monitoring of human physiological data, in both normal and abnormal situations of activity, is interesting for the purpose of emergency event detection, especially in the case of elderly people living on their own. Several techniques have been proposed for identifying such distress situations using either motion, audio or video data from the monitored subject and the surrounding environment. This paper aims to present an integrated patient fall detection platform that may be used for patient activity recognition and emergency treatment. Both visual data captured from the user's environment and motion data collected from the subject's body are utilized. Visual information is acquired using overhead cameras, while motion data is collected from on-body sensors. Appropriate tracking techniques are applied to the aforementioned visual perceptual component enabling the trajectory tracking of the subjects. Acceleration data from the sensors can indicate a fall incident. Trajectory information and subject's visual location can verify fall and indicate an emergency event. Support Vector Machines (SVM) classification methodology has been evaluated using the latter acceleration and visual trajectory data. The performance of the classifier has been assessed in terms of accuracy and efficiency and results are presented.
\end{abstract}

\section{Introduction}

The telemonitoring of human physiological data, in both normal and abnormal situations of activity, is interesting for the purpose of emergency event detection or long term data-storage for later diagnosis or for the purpose of medical exploration. In the case of elderly people living on their own, there is a particular need for monitoring their behavior. The goal of this surveillance is the detection of major

Please use the following format when citing this chapter:

Doukas, C., Maglogiannis, I., Katsarakis, N. and Pneumatikakis, A., 2009, in IFIP International Federation for Information Processing, Volume 296; Artificial Intelligence Applications and Innovations III; Eds. Iliadis, L., Vlahavas, I., Bramer, M.; (Boston: Springer), pp. 185-193. 
incidents such as a fall, or a long period of inactivity in a part of their area. Several techniques have been proposed for identifying such distress situations using either motion, audio or video data from the monitored subject and the surrounding environment. This paper presents a human body fall detection platform based both motion and visual perceptual components. A number of on-body sensors collect the movement data and transmit them wirelessly to the monitoring unit, while overhead cameras track the trajectory and shape of the body and provide information regarding the patient's position and activity. Appropriate classification of the motion data can give an indication of a fall. Combining the latter with unusual change of body' shape followed by inactivity, an alarm can be triggered and more information regarding the severity of the incident can be obtained; in case patient remains still after the fall or moves but the body is detected on the ground then the patient requires immediate assistance.

The rest of the paper is organized as follows; Section 2 discusses related work in the context of patient activity and fall detection. Section 3 describes the proposed system architecture and Sections 4 and 5 describe the acquisition of the patient movement and visual data using sensors and overhead cameras respectively. Section 6 presents the data classification using Support Vector Machines and corresponding evaluation results and finally Section 7 concludes the paper.

\section{Related Work}

Although the concept of patient activity recognition with focus on fall detection is relatively new, there exists related research work, which may be retrieved from the literature ([1]-[9]). Information regarding the patient movement and activity is frequently acquired through visual tracking of the patient's position. In [5] overhead tracking through cameras provides the movement trajectory of the patient and gives information about user activity on predetermined monitored areas. Unusual inactivity (e.g., continuous tracking of the patient on the floor) is interpreted as a fall. Similarly, in 8 omni-camera images are used to determine the horizontal placement of the patient's silhouettes on the floor (case of fall). Success rate for fall detection is declared at $81 \%$ for the latter work. A different approach for collecting patient activity information is the use of sensors that integrate devices like accelerometers, gyroscopes and contact sensors. The latter approach is less depended on the patient and environmental information and can be used for a variety of applications for user activity recognition ([1], [3], [7]). Regarding fall detection, authors in [2], [6], [9] use accelerometers, gyroscopes and tilt sensors for movement tracking. Collected data from the accelerometers (i.e., usually rotation angle or acceleration in the $\mathrm{X}, \mathrm{Y}$ and $\mathrm{Z}$ axis) is used to verify the placement of the patient and time occupation in rooms and detect abrupt movement that could be associated with fall. Detection is performed using predefined thresholds [1], [3], [4], [6] and association between current position, movement and acceleration [2], [9]. To our best knowledge there is no work in the literature that combines both visual 
and sensor information for a more complete and robust estimation of a patient's fall and can provide some information regarding the severity of the incident (e.g. patient has gotten up right after the fall, patient is inactive, etc.).

\section{System Architecture Overview}

The presented system follows the architecture illustrated in Fig. 1. Accelerometers data are collected through the sensor attached on the user's chest and belt and are transmitted wirelessly to the monitoring node. Transmission of data is performed through J2ME sockets following the client-server architecture. The monitoring unit acting as movement data receiver serves as the server whereas each node is the client.

At the same time, camera devices record video frames from the user's site and provide feed to the video tracker. The latter tracks the movement of the patient's body and generates body shape features (i.e. coordinates of a bounding box containing the subject's body). The data are properly transformed in a suitable format for the classifier and the classification phase begins. Based on a predefined classification model (i.e. train model), the patient status is detected (i.e. emergency status when fall detected, normal status otherwise).

Apart from the indication of a fall incident, an estimation of the severity of the incident can be provided based on the patient's behavior after the fall as recorded visually; movement indicated by accelerometers but visual inactivity or soft activity suggests that patient has not lost consciousness and is trying to recover from the fall, both sensor and normal visual activity can indicate that patient has recovered from fall, and no activity at all can indicate higher severity of the incident.

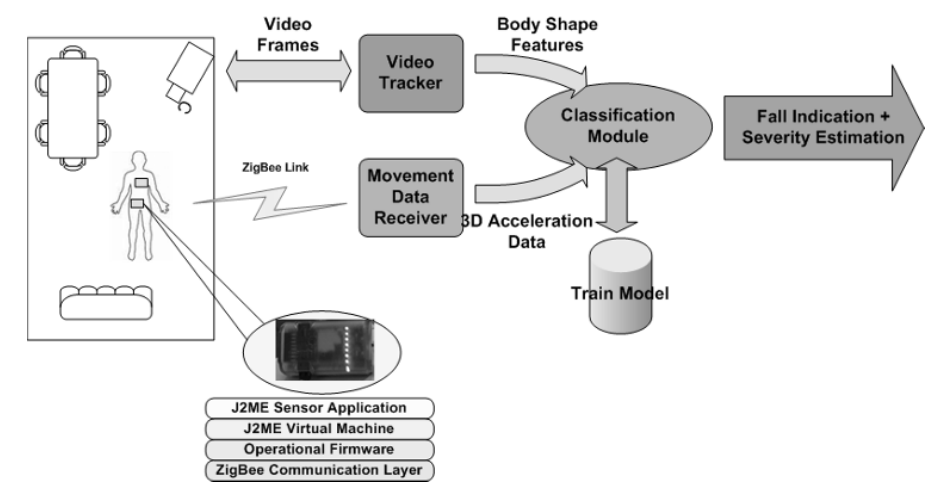

Fig. 1. Platform Architecture and Data interaction between the movement capturing tools and monitoring node. 


\section{Patient Movement Data Acquisition}

This section provides information on the acquisition and pre-processing of the patient movement data. The Sentilla Perk [10] sensor kit has been utilized in our system. The latter contains two $2.4 \mathrm{GHz}$ wireless data transceivers (nodes, see Fig. 2) using the IEEE 802.15.4 (ZigBee) protocol. It also includes a USB port for interface with a personal computer acting as the monitoring unit. Each node has a lowpower, low-voltage MCU (MicroController Unit), one 3D Accelerometer for X, Y and $\mathrm{Z}$ axis and additional analog and digital input pins for adding more sensors. The Perk nodes are provided in a plastic robust small-sized enclosure $(6 \times 3 \times 1.5 \mathrm{~cm})$ making them more suitable for placing on patient's body and tolerating falls.

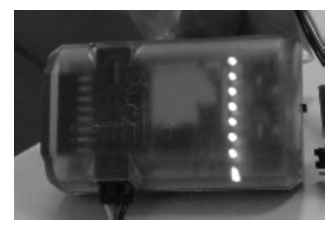

(a)

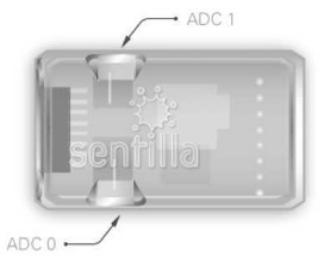

(b)

Fig. 2. The Sentilla Perk node containing a 3D accelerometer that can be attached on user and send motion data through the ZigBee wireless protocol. The plastic enclosure can protect the node from falls and makes it more suitable for carrying it on patient's body. A) Actual photo of the node, b) illustration indicating two analog-to-digital converter ports for the addition of alternative sensors.

Two Perk nodes can be placed on patient's body. Preferable positions are close to user's chest and user's belt or lower at user's foot. The latter positions have proven based on conducted experiments to be appropriate for distinguishing rapid acceleration on one of the three axis that is generated during a fall.

Appropriate J2ME [17] code is developed and deployed on the nodes for reading the accelerometer values and transmitting them wirelessly to the monitoring unit. At the latter a Java application built using the Sentilla IDE [10] receives the movement data and performs further processing as described in the following sections. An example of motion data as received by the two sensor nodes is illustrated in Fig. 3. The X, Y and $\mathrm{Z}$ acceleration values from both sensors are interlaced.

\section{Video Tracking of Human Body}

The goal of the developed body video tracker is to provide across time the frame regions occupied by human bodies. The tracker is built around a dynamic foreground segmentation algorithm [12] that utilizes adaptive background modeling. This is based on Stauffer's algorithm [13] to provide the foreground pixels. 
Stauffer's algorithm models the different colors every pixel can receive in a video sequence by Gaussian Mixture Models (GMM). One GMM corresponds to every pixel at given coordinates across time. The Gaussians are three-dimensional, corresponding to the red, green and blue components of the pixel color. Their weight is proportional to the time a particular Gaussian models best the color of the pixel. Hence the weight of a given Gaussian is increased as long as the color of the pixel can be described by that Gaussian with higher probability than any other Gaussian in the GMM can, and that probability is above a threshold. As a result, a map can be built in which every pixel is represented by the weight of the Gaussian from its GMM that best describes its current color. This is the Pixel Persistence Map (PPM): Regions of the map with large values correspond to pixels that have colors that appear there for a long time, hence they belong to background. On the contrary, regions with small values correspond to pixels that have colors that appear there for a short time, hence they are foreground. This is true as long as the foreground objects have distinct colors from the background.

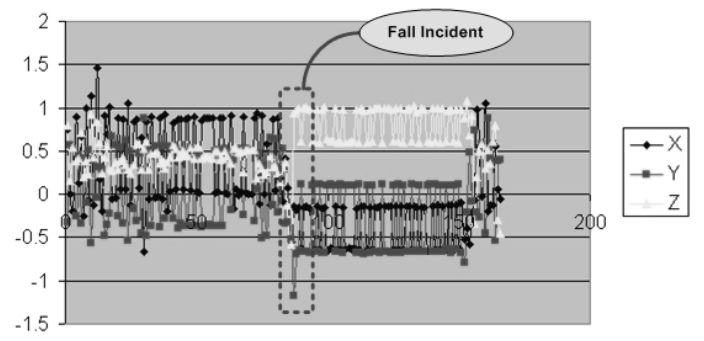

Fig. 3. Illustration of interlaced from both sensors acceleration data in $\mathrm{X}, \mathrm{Y}$ and $\mathrm{Z}$ axis. The $\mathrm{Y}$ axis represents the acceleration value (range between -2 and 2) and the $X$ axis the number of samples acquired.

The problem of Stauffer's algorithm is with foreground objects that stop moving. In its original implementation, targets/objects that stop moving are learnt into the background. This happens as the weights of the Gaussians of the GMM of pixels describing the foreground colors and corresponding to immobile foreground objects increase with time. To avoid this, the learning rates of the adaptation that increase the weights of Gaussians are not constant, neither across space, nor across time. Instead, they are spatiotemporally controlled by the states of Kalman filters [11]. Every foreground area corresponds to a target being tracked by a Kalman filter. The foreground pixels are combined into body evidence blobs, used for the measurement update stage of the Kalman filters. The states are used to obtain the position, size and mobility of each target, the latter being a combination of translation and size change. This information is fed back to the adaptive background modeling module to adapt the learning rate in the vicinity of each target: frame regions that at a specific time have a slow-moving target have smaller learning rates. The block diagram of the body tracker is shown in Fig. 4. 
With the feedback configuration of the tracker, the learning of the slow moving foreground objects into the background is slowed down long enough for the intended application, i.e. tracking people moving indoors and possibly falling down. The tracker results when applied on the visual feed by an overhead camera are illustrated in Fig. 5.

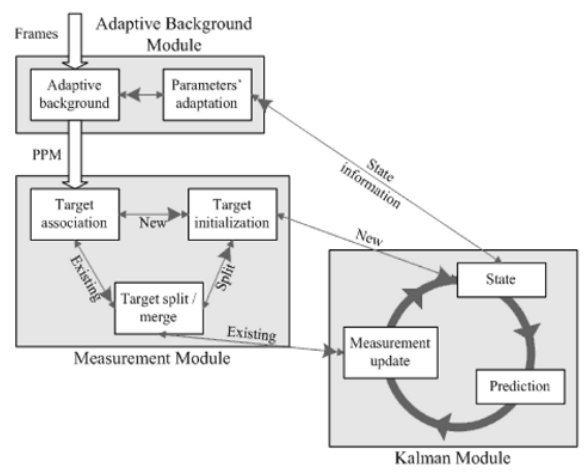

Fig. 4. Block diagram of the body video tracker. Kalman filters spatiotemporally adapt the learning rates of the adaptive background algorithm, effectively avoiding learning of immobile foreground objects into the background.

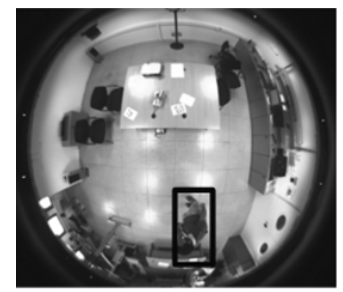

A

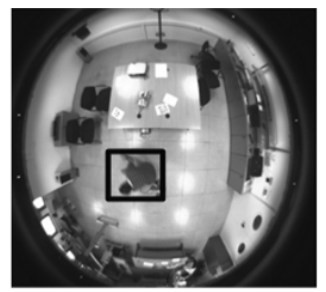

B

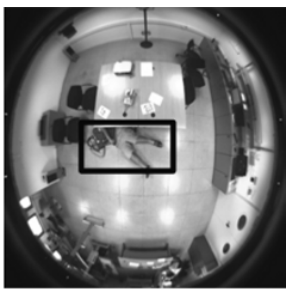

C

Fig. 5. Visualization of video tracking performance. The tracker detects the movement of the body and correlates it with the movement of a rectangular blob within the visual domain. Upper left $\mathrm{X}, \mathrm{Y}$ coordinates and respective width and height of the blob are reported for each visual frame. Frame A corresponds to normal walking, Frame B to captured movement during fall and Frame $\mathrm{C}$ illustrates detection of body in horizontal position after fall.

Tracking through overhead cameras has been selected due to the fact that it provides a better visual representation of the monitored area and allows the tracker to gain a better estimation of the body shape when subject moves, falls and lays still after fall. The presented tracker creates and tracks a rectangular blob around the detection of the moving body within the frames and reports the upper left corner coordinates and respective width and height of the blog. As indicated in Fig. 5 the size of the blob changes during the fall and after it. 


\section{The System in Practice: Classification of Motion and Visual Perceptual Components}

This Section provides information regarding the classification method used and reports the accuracy of the system in the detection of a patient fall. According to our previous research [14], [15] the SVM (Support Vector Machines) classification method has been proved to obtain high accuracy in the detection of fall incidents based on movement data. More particularly accuracy rates for the distinction of fall against other movement types can reach $98.2 \%$. In previous experiments the train model has been built using only acceleration data whereas in the proposed system the train model contains also visual information as described in Section 5. The WEKA tool [16] has also been used for the development and evaluation the SVM model. Classification data are provided in the following form:

$$
\text { Fall_ID } X \quad Y \quad Z \quad B B x \quad B B y \quad B B_{\text {Width }} \quad B B_{\text {Height }}
$$

where $\mathrm{X}, \mathrm{Y}$ and $\mathrm{Z}$ are the acceleration data as retrieved from the sensors, BBx and BBy are the upper left coordinates of the bounding box that tracks patient's body and BBwidth and BBheight the width and height of the bounding box respectively. Fall_ID represents the case of fall incident (true or false).

To evaluate the efficiency and accuracy of the presented platform in the context of detecting patient falls, a number of experiments were conducted; a volunteer wearing the sensors devices described in Section 4 was recorded walking and falling in different locations and ways while an overhead camera was capturing visual frames. Motion data and body shape features are utilized for creating classification models. The 10-cross fold validation methodology has been used to verify each model's accuracy and performance.

Apart from the detection of fall the system is also capable of estimating the severity of the incident: When an estimation of a fall has occurred based on the sensor and visual data the standard deviation of accelerometer values and visual bounding box values is calculated for the next 15 seconds. A specific threshold has been determined for each value that can determine the severity of the incident according to the following table:

Table 1. Decision matrix for the severity of a fall incident based on standard deviations of movement data and body bounding box coordinates after a fall has occurred.

\begin{tabular}{|l|l|l|}
\hline $\begin{array}{l}\text { Motion } \\
\text { STD }\end{array}$ & $\begin{array}{l}\text { Bounding Box }(\mathrm{X}, \mathrm{Y}) \\
\text { STD }\end{array}$ & Severity \\
\hline$>0.5$ & $>60$ & Low. Patient has recovered from fall (gotten up) \\
\hline$>0.5$ & $<60$ & $\begin{array}{l}\text { Medium. Patient is moving but cannot fully recover } \\
\text { from fall }\end{array}$ \\
\hline$<0.5$ & $<60$ & $\begin{array}{l}\text { High. No activity is recorded; patient has probably } \\
\text { felt unconscious }\end{array}$ \\
\hline
\end{tabular}


Table 2. Accuracy evaluation results of the proposed System. Motion and video tracking data of four fall experiments have been used. Percentage of correctly classified results, Root Mean Squared Error and correctly classified severity of the fall are presented.

\begin{tabular}{|l|l|l|l|l|}
\hline Experiment & $\begin{array}{l}\text { Correctly Clas- } \\
\text { sified Fall (\%) }\end{array}$ & $\begin{array}{l}\text { Root Mean } \\
\text { Squared Error }\end{array}$ & $\begin{array}{l}\text { Interlaced Mo- } \\
\text { tion Data }\end{array}$ & $\begin{array}{l}\text { Correctly Clas- } \\
\text { sified Severity }\end{array}$ \\
\hline FallA & 99.2 & 0.0112 & Yes & Yes \\
\hline FallB & 100.0 & 0.0072 & Yes & Yes \\
\hline FallC & 99.4 & 0.0082 & Yes & Yes \\
\hline FallD & 98.7 & 0.0121 & Yes & Yes \\
\hline FallA & 97.3 & 0.0242 & No & Yes \\
\hline FallB & 98.4 & 0.0173 & No & Yes \\
\hline FallC & 97.1 & 0.0449 & No & Yes \\
\hline FallD & 96.9 & 0.0534 & No & Yes \\
\hline
\end{tabular}

According to the evaluation results as presented in Table 2, the SVM seem to achieve high accuracy rates in all cases. When the motion data from both on-body sensors are interlaced accuracy proves to be higher than otherwise. Finally, the severity of each fall incident is correctly estimated in all cases based on the motion and video track data after the fall.

\section{Conclusions}

In this paper an enhanced patient fall detection system has been proposed that combines both motion and visual information. Accelerometer data obtained through wireless sensors in conjunction to body shape features acquired by visual tracking are evaluated through a SVM train model. A detection of a fall incident is then generated. In addition, combining the motion data and movement of the body obtained visually after the fall, the severity of the fall can also be estimated alerting treatment personnel appropriately.

\section{References}

1. Noury N., Herve T., Rialle V., Virone G., Mercier E., Morey G., Moro A., Porcheron T., "Monitoring behavior in home using a smart fall sensor and position sensors", In Proc. 1st Annual International Conference on Microtechnologies in Medicine and Biology, pp. 607-610, Oct. 2000.

2. Noury N., "A smart sensor for the remote follow up of activity and fall detection of the elderly", In Proc. 2nd Annual International Conference on Microtechnologies in Medicine and Biology, pp. 314-317, May 2002.

3. Prado M., Reina-Tosina J., Roa L., "Distributed intelligent architecture for falling detection and physical activity analysis in the elderly", In Proc. 24th Annual IEEE EMBS Conference, pp. 1910-1911, Oct. 2002. 
4. Fukaya K., "Fall detection sensor for fall protection airbag", In Proc. 41st SICE Annual Conference, pp. 419-420, Aug. 2002.

5. Nait-Charif, H. McKenna, S.J., "Activity summarisation and fall detection in a supportive home environment”, In Proc. 17th International Conference on Pattern Recognition ICPR 2004, pp. 323-236, Aug. 2004.

6. Hwang, J.Y. Kang, J.M. Jang, Y.W. Kim, H.C., "Development of novel algorithm and real-time monitoring ambulatory system using Bluetooth module for fall detection in the elderly", In Proc. 26th Annual International Conference of the IEEE Engineering in Medicine and Biology Society, pp. 2204-2207, 2004.

7. Shuangquan Wang, Jie Yang, Ningjiang Chen, Xin Chen, Qinfeng Zhang, "Human activity recognition with user-free accelerometers in the sensor networks", In Proc. International Conference on Neural Networks and Brain, pp. 1212-1217, Oct. 2005.

8. S.-G. Miaou, Pei-Hsu Sung, Chia-Yuan Huang, "A Customized Human Fall Detection System Using Omni-Camera Images and Personal Information", In Proc. 1st Transdisciplinary Conference on Distributed Diagnosis and Home Healthcare, pp.3942, 2006.

9. Allen, F.R. Ambikairajah, E. Lovell, N.H. Celler, B.G., "An Adapted Gaussian Mixture Model Approach to Accelerometry-Based Movement Classification Using TimeDomain Features", In Proc. 28th Annual International Conference of the IEEE Engineering in Medicine and Biology Society, pp. 3600-3603, Aug. 2006.

10.The Sentilla Perk Pervasive Computing Kit, http://www.sentilla.com/perk.html

11.R. E. Kalman, "A New Approach to Linear Filtering and Prediction Problems", Transactions of the ASME - Journal of Basic Engineering, Vol.82, Series D, pp.35-45, 1960.

12.Pnevmatikakis and L. Polymenakos, "Robust Estimation of Background for Fixed Cameras," International Conference on Computing (CIC2006), Mexico City, Mexico, 2006.

13.Stauffer and W. E. L. Grimson, "Learning patterns of activity using real-time tracking," IEEE Transactions on Pattern Analysis and Machine Intelligence, Vol. 22, No. 8, pp. 747-757, 2000.

14. Charalampos Doukas, Ilias Maglogiannis, Philippos Tragkas, Dimitris Liapis, Gregory Yovanof, "Patient Fall Detection using Support Vector Machines", In Proc. 4th IFIP Conference on Artificial Intelligence Applications \& Innovations (AIAI), Sept. 19-21, Athens, Greece.

15.Charalampos Doukas, Ilias Maglogiannis, "Advanced Patient or Elder Fall Detection based on Movement and Sound Data", presented at 2nd International Conference on Pervasive Computing Technologies for Healthcare 2008.

16. Ian H. Witten and Eibe Frank (2005) "Data Mining: Practical machine learning tools and techniques", 2nd Edition, Morgan Kaufmann, San Francisco, 2005.

17.The JAVA ME Platform, http://java.sun.com/javame/index.jspf 